\title{
Fast wave current drive in DEMO
}

\author{
E. Lerche ${ }^{\mathrm{a}}$, D. Van Eester ${ }^{\mathrm{a}}$, A. Messiaen ${ }^{\mathrm{a}}$, T. Franke ${ }^{\mathrm{b}}$ and EFDA-PPPT contributors \\ ${ }^{a}$ Association EURATOM-Belgian State, LPP-ERM/KMS, TEC partner, Brussels, Belgium \\ ${ }^{b}$ Max-Planck-Institut für Plasmaphysik, EURATOM-Assoziation, Garching, Germany
}

\begin{abstract}
The ability to non-inductively drive a large fraction of the toroidal plasma current in magnetically confined plasmas is an essential requirement for steady state fusion reactors such as DEMO. Besides neutral beam injection (NBI), electron-cyclotron resonance heating (ECRH) and lower hybrid wave heating ( $\mathrm{LH})$, ion-cyclotron resonance heating (ICRH) is a promising candidate to drive current, in particular at the high temperatures expected in fusion plasmas. In this paper, the current drive (CD) efficiencies calculated with coupled ICRF wave / CD numerical codes for the DEMO-1 design case $\left(R_{0}=9 \mathrm{~m}, B_{0}=6.8 \mathrm{~T}, a_{p}=2.25 \mathrm{~m}\right)$ [1] are presented. It will be shown that although realistic CD efficiencies can be obtained in the usual ICRF frequency domain $(20-100 \mathrm{MHz})$ by shifting the dominant ion-cyclotron absorption layers to the high-field side, operation at higher frequencies $(100-300 \mathrm{MHz})$ has a stronger CD potential, provided the parasitic RF power absorption of the alpha particles can be minimized.
\end{abstract}

Keywords: ICRF, current-drive, tokamak, DEMO

PACS: 52.50.Qt, 52.55.Wq, 52.55.Fa, 28.52.Cx

\section{INTRODUCTION}

The local current drive efficiencies $\chi_{\mathrm{CD}}(\rho)=J_{\mathrm{e}} / P_{\mathrm{RF}, \mathrm{e}}$ (with $\rho$ being the minor radius) provided in this work rely on the expression due to Ehst [2], $\chi_{\mathrm{CD}}(\rho)=38.4 \times 10^{18}\left(T_{\mathrm{e}} / n_{\mathrm{e}}\right) \eta_{\text {Ehst }} / \ln \Lambda$, where $\ln \Lambda$ is the Coulomb logarithm and $\eta_{\text {Ehst }}$ is a function of $Z_{\text {eff }}$ (effective charge), $\varepsilon=\rho / R$ (orbit effects) and the Ehst parameter $w=\omega /\left(k_{/}, N_{\mathrm{te}}\right)$, the ratio between the phase velocity of the waves $\omega / k_{/ /}$and the local thermal velocity of the electrons $\mathrm{v}_{\mathrm{te}}=\left(k_{\mathrm{B}} T_{\mathrm{e}} / m_{\mathrm{e}}\right)^{1 / 2}$. For parameters for which current drive is efficient, the local CD efficiency is roughly proportional to $w^{2}$ and thus higher frequencies and/or lower parallel wave numbers $k_{/ /}=N_{\phi} / R$ (where $N_{\phi}$ is the toroidal mode number imposed by the antenna spectrum), privilege efficient non-inductive current-drive. The expression given by Ehst allows estimating the current driven for a given electron absorption profile but it does not say anything about the efficiency of the electron power absorption itself. Opposite to CD efficiency, electron Landau damping $P_{\mathrm{RF}, \mathrm{e}}$ is most efficient when the parallel wave phase velocity matches the thermal velocity i.e. $w=\omega /\left[k_{/} / \mathrm{V}_{\mathrm{te}}\right] \sim 1$. Since both the electron power absorption and the current-drive processes need to be efficient to guarantee a significant net current per MW launched, a reasonable compromise between these 2 requirements needs to be found. As the absorbed power density falls exponentially away from $w \sim 1$ while the current drive efficiency is only increasing roughly quadratically, a good compromise is to operate at modest w slightly exceeding unity. The increase of the current drive efficiency $J_{\mathrm{e}} / P_{\mathrm{RF}, \mathrm{e}}$ at large $w$ is somewhat misleading since a prerequisite for current drive is a suitable absorption of the wave energy by the electrons and so the overall driven current may be modest if $P_{\mathrm{RF}, \mathrm{e}}$ is small, in spite of the promising value of $\chi_{\mathrm{CD}}$. To ensure good electron absorption, the ion cyclotron absorption of the different plasma constituents must me minimized in the different frequency domains, as will be show in the next 2 sections. The fact that the parameter $\mathrm{w}$ depends on the temperature reveals that the optimal choice to maximize the driven current forces one to adapt $\omega / k_{/ /}$for a given - projected - temperature.

\section{STANDARD ICRF FREQUENCY DOMAIN (20-100MHZ)}

In the usual ICRF frequency domain $(f<100 \mathrm{MHz})$, electron absorption is maximized by avoiding efficient cyclotron absorption of the main $\mathrm{D}$ and $\mathrm{T}$ bulk ions. Figure 1 shows the integrated RF power absorption as function of the RF frequency as computed by the 1D TOMCAT wave code [3] for a plasma composed of equal amounts of D and T, $1 \%$ of fast fusion born $\alpha$-particles (Maxwellians at 1.2keV), $10 \%$ thermal $\alpha$ 's and traces of Be and Ar. One can immediately identify 2 regions of dominant electron absorption with potential for efficient CD: $f<25 \mathrm{MHz}$, where none of the plasma ions is resonant inside the plasma and $f=70-75 \mathrm{MHz}$, where the second harmonic $\mathrm{T}$ absorption is shifted to the high-field side and the harmonic resonances of $\mathrm{D}$ and $\alpha$-particles have not yet entered the plasma. 


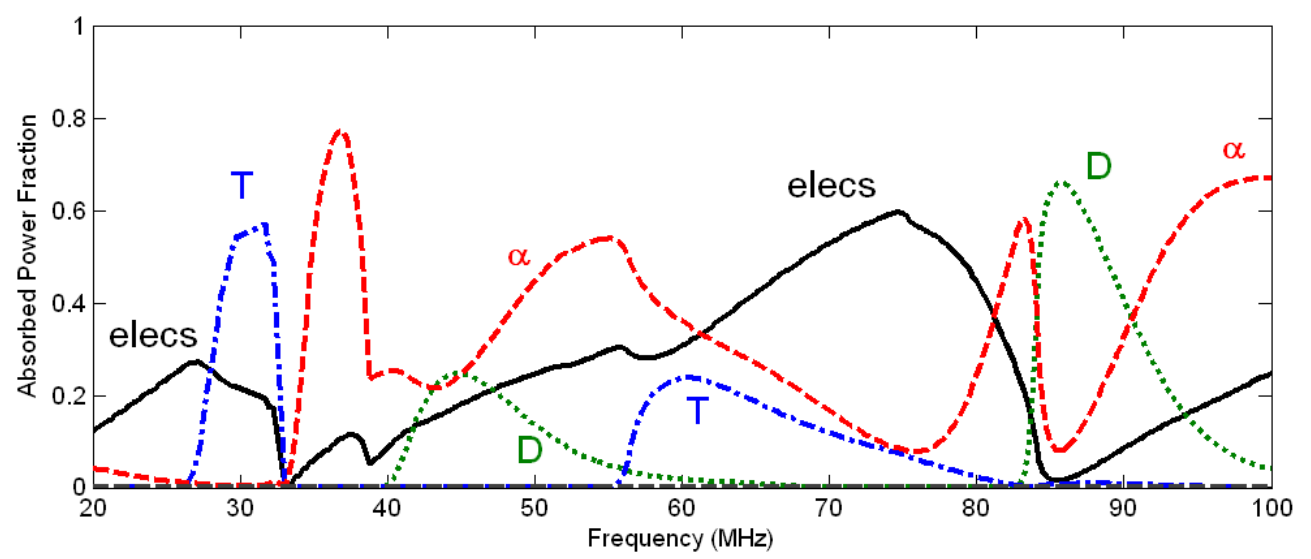

FIGURE 1. Absorbed power fractions vs. frequency for the DEMO-1 C case $\left(n_{\mathrm{e} 0}=1.0 \times 10^{20} / \mathrm{m}^{3}, T_{\mathrm{e} 0}=29 \mathrm{keV}\right)$ for $N_{\phi}=80$.

Fig. 2a illustrates the total CD efficiency $\eta_{\mathrm{CD}}=\int_{\chi \mathrm{CD}}(\rho) \mathrm{dS}$ as function of the toroidal wave number $N_{\phi}$ as computed by the 2D full-wave CYRANO code [4] for 3 cases: $f=25 \mathrm{MHz}$ (low frequency CD scheme), $f=64 \mathrm{MHz}$ (heating scenario) and $f=72 \mathrm{MHz}$ (up-shifted frequency CD scheme). Fig. 2c shows the integrated currents per Watt launched as function of minor radius for the three cases when convoluting the CD efficiency values shown in Fig.2a with the standard CD $(0 \pi / 2 \pi 3 \pi / 2)$ antenna spectrum shown in Fig.2b (solid line), calculated with the ANTITER code [5] for an antenna identical to the one being designed for ITER but placed in DEMO $\left(R_{\mathrm{ant}}=11.45 \mathrm{~m}\right)$. One readily sees that the $f=72 \mathrm{MHz}$ case gives the highest $\mathrm{CD}$ value $(0.037 \mathrm{~A} / \mathrm{W})$ with a maximum driven current around $\rho=0.5-0.8 \mathrm{~m}$, followed by the $f=25 \mathrm{MHz}$ case $(0.032 \mathrm{~A} / \mathrm{W})$, which features more central current-drive. As expected, the heating case $(f=64 \mathrm{MHz})$ offers low CD capabilities, since the tritons absorb the majority $(\sim 2 / 3)$ of the ICRF power.
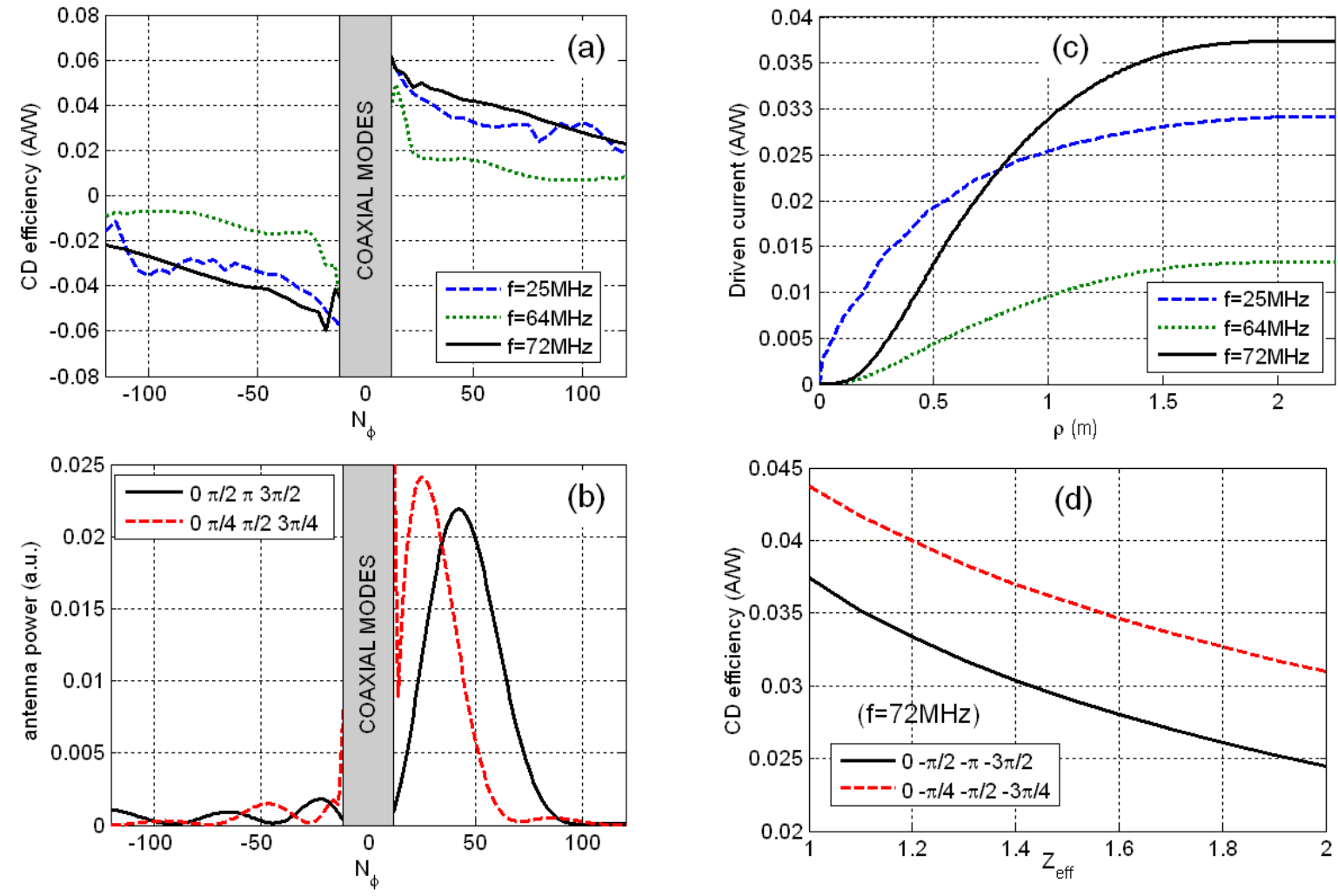

FIGURE 2. (a) CD efficiencies as function of $N_{\phi}$ for 3 cases: $f=25 \mathrm{MHz}$ (dashed), $f=64 \mathrm{MHz}$ (dotted) and $f=72 \mathrm{MHz}$ (solid); (b) ITER-like toroidal antenna spectrum for 2 phasing configurations; (c) Integrated driven currents per W launched for the 3 frequencies analyzed; (d) CD efficiencies as function of $Z_{\text {eff }}$ for the 2 antenna phasings shown in $b$. 
The effect of the plasma composition on the CD efficiency is illustrated in Fig.2d, where $\eta_{\mathrm{CD}}$ is plotted against the effective charge $Z_{\text {eff. }}$ It is seen that there is a substantial reduction in the CD efficiency with $Z_{\text {eff }}$ due to the increased plasma resistivity and for the nominal DEMO value of $Z_{\mathrm{eff}}=1.6$, the efficiency using standard $\pi / 2 \mathrm{CD}$ phasing is reduced from 0.037 to $0.028 \mathrm{~A} / \mathrm{W}$. By using an antenna phasing that privileges the excitation of lower $\mathrm{k}_{/ /}$ modes (as the $\pi / 4$ example shown in Fig. $2 \mathrm{~b}$ - dashed) or by designing a wider antenna that by definition launches low $k_{/ /}$waves, this value can be increased [6].

\section{HIGH FREQUENCY DOMAIN (100-300MHZ)}

Current drive becomes more efficient at higher frequencies and as thermal populations are poor RF power absorbers at high cyclotron harmonics, it makes sense to explore the potential of FWCD at frequencies beyond the range considered so far. An evident obstacle is the fact that very energetic fusion-born $\alpha$ 's (here modeled as $1.2 \mathrm{MeV}$ Maxwellians) compete strongly with electron heating as various cyclotron layers reside simultaneously in the plasma and ion heating at each of them is non-negligible. Figs 3a and $3 b$ illustrate the competition between electron and alpha particle absorption for two different $k_{/ /}$values when the RF frequency is increased towards high frequencies. Figs $3 c$ and $3 d$ show the corresponding CD efficiencies $\eta_{C D}$. One immediately sees that from the electron absorption point of view, using waves with higher $k_{/ /}$(b) is more favorable since the alpha particle absorption is somewhat reduced. However, the $k_{/ /}$values that are necessary to guarantee substantial electron absorption are too high for efficient current drive (d), since the increase in $k_{/ /}$cancels the beneficial effect of the increase in frequency in the Ehst parameter $w$, leading to $\eta_{\mathrm{CD}}$ values that are (even) lower than the ones obtained in the standard frequency case $(72 \mathrm{MHz})$. The oscillations in the power absorption values represent the shifting of the harmonic alpha particle ion-cyclotron resonance layers in the plasma when the frequency is scanned.
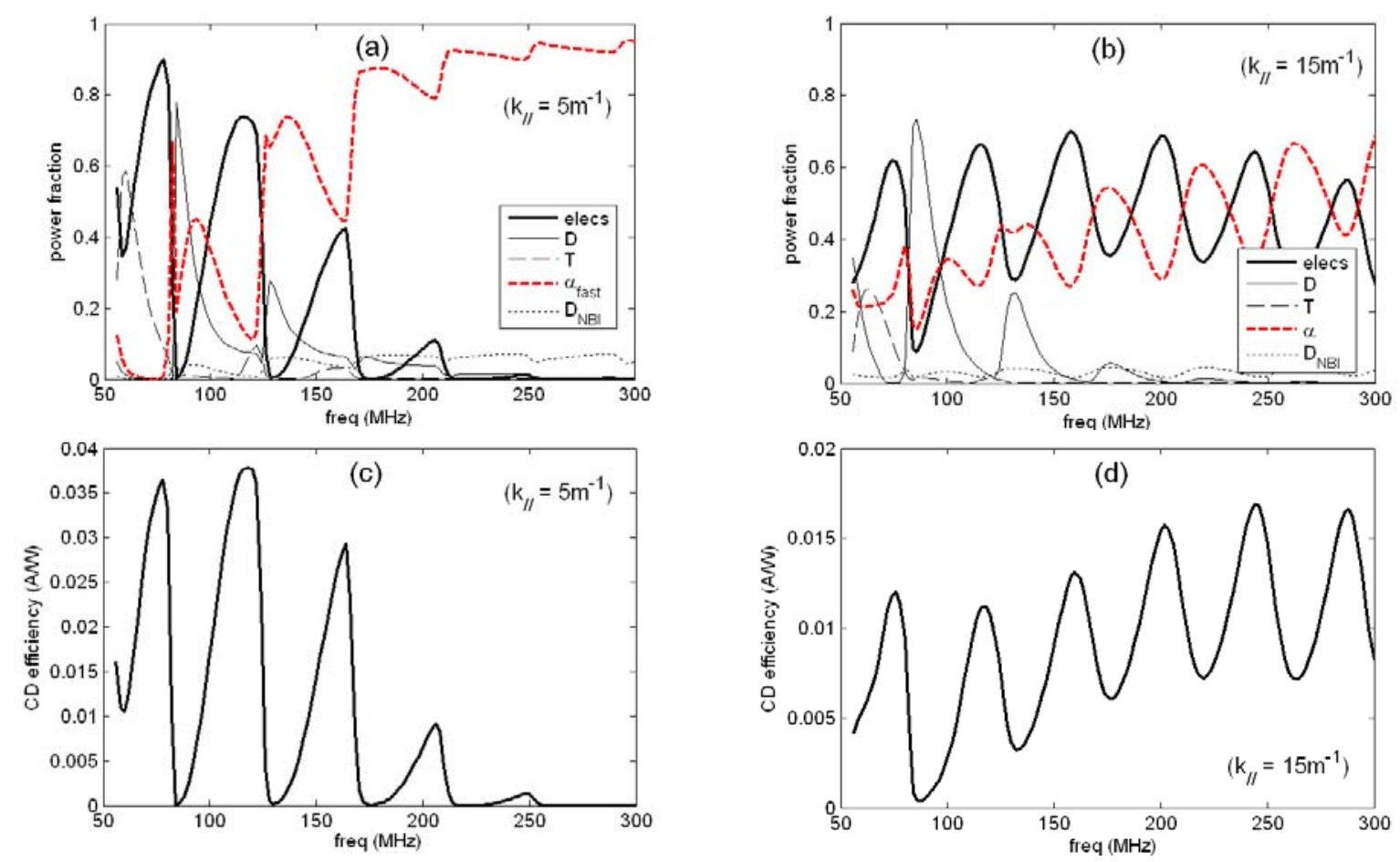

FIGURE 3. (a,b) Absorbed power fractions of various species as function of frequency for $k_{/ /}=5 \mathrm{~m}^{-1}$ and $k_{/ /}=15 \mathrm{~m}^{-1}$; (c,d) Respective CD efficiency values as function of frequency.

One alternative to minimize $\alpha$-particle absorption when launching low $k_{/ /}$waves at high frequencies could be to use antennas that launch the waves from the top of the machine, in-between the harmonic cyclotron layers of the $\alpha$ particles [7]. Figs. 4a and 4b show the potential of such approach, and illustrate respectively, the power fraction absorbed by the electrons and the CD efficiency as function of frequency for the standard case (EQUAT) and for the top launch case (TOP), which uses the same antenna but it is now located at the top of the tokamak centered at $R=R_{0}$, for $k_{/ /}=5 \mathrm{~m}^{-1}$ rather than in the equatorial plane. Although the oscillations corresponding to the shifting of the 
$\alpha$-particle cyclotron layers is also present in the top launch case, one sees that the electron absorption reaches $100 \%$ when the $\alpha$ cyclotron harmonics are not on-axis. In these operational windows, the beneficial effect of operating at high frequencies is clearly reflected in the CD efficiency and the $\eta_{C D}$ values increase strongly with frequency reaching exceptionally high values $\left(\eta_{\mathrm{CD}}>0.1 \mathrm{MA} / \mathrm{MW}\right)$. Notwithstanding the technical difficulties of a top launch antenna design, these results encourage a deeper assessment of this option for fusion reactor application, since it can enhance the standard frequency range FWCD efficient by more than a factor of three.
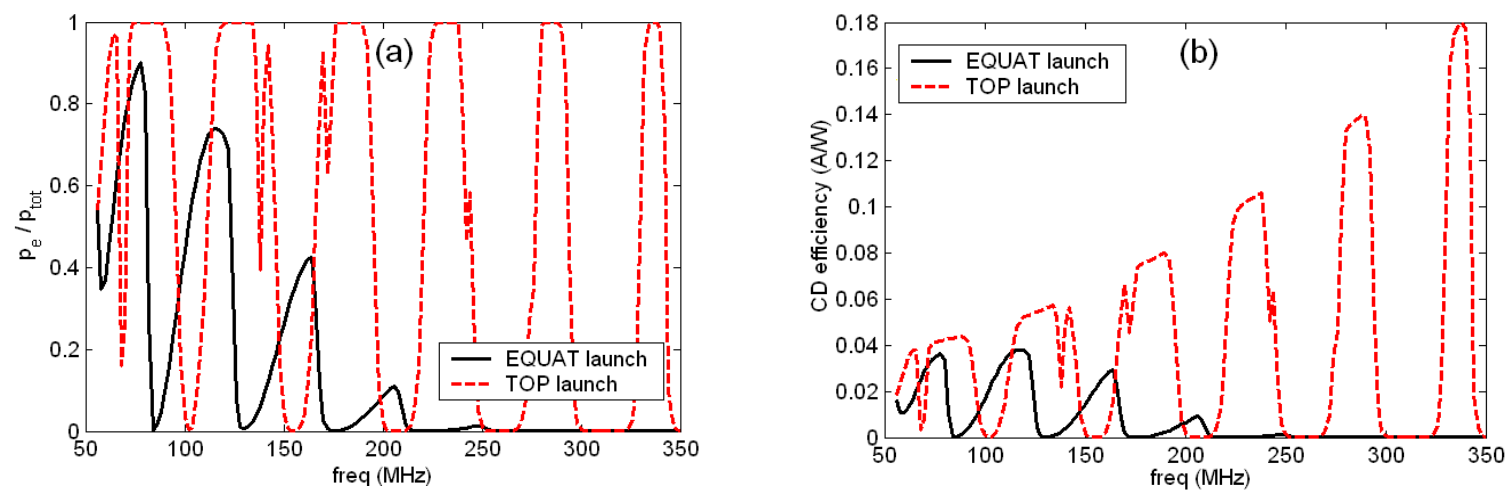

FIGURE 4. (a) Electron power absorption fraction and (b) current-drive efficiency as function of frequency for the equatorial launch (EQUAT) and top launch (TOP) cases for $k_{/ /}=5 \mathrm{~m}^{-1}$.

\section{SUMMARY}

The standard ICRH frequency range offers two windows for efficient non-inductive current-drive in DEMO: $f<20-25 \mathrm{MHz}$ and $f=70-75 \mathrm{MHz}$. Because the latter features higher antenna coupling, higher single-pass wave absorption and higher CD efficiency, this scheme is recommended as the main ICRF current-drive scenario for DEMO. Moreover, because its frequency is closer to the one to be used in the heating scenario $(f=64 \mathrm{MHz})$, the RF system could easily be designed to cover both frequencies and one could think of a real-time frequency control that allows ICRF heating until the discharge reaches fusion dominant heating and subsequent change to current-drive for steady-state operation without switching-off the ICRF power. Although the CD efficiency depends on the plasma temperature (due to both the electron power absorption and the plasma resistivity), the actual antenna spectrum (dictated by the antenna geometry and the strap phasing) is an important parameter: Pure anti-symmetric spectra and low $k_{/ /}=N_{\phi} / R$ excitation privileges efficient current drive. For $f=72 \mathrm{MHz}$, the CD efficiency values for the nominal DEMO value of $Z_{\mathrm{eff}}=1.6$ are between $\eta_{\mathrm{cd}}=0.028-0.035 \mathrm{~A} / \mathrm{W}$ depending on the antenna phasing used, which translates into a normalized efficiency of $\eta=n_{\mathrm{e}, 0} R_{0} \eta_{\mathrm{cd}}=0.26-0.32 \mathrm{~A} / \mathrm{W} / \mathrm{m}^{2}$ for the density and geometry considered in the simulations $\left(n_{\mathrm{e}, 0}=10^{20} / \mathrm{m}^{3}, R_{0}=9.2 \mathrm{~m}\right)$.

Whereas the ICRF current drive efficiency is modest in the usual frequency range, it can be enhanced significantly when operating at high frequency. However, parasitic alpha particle heating (whose high harmonic IC resonance absorption is efficient) hinders optimal electron absorption and should be avoided. In the standard (equatorial) launch case, $\alpha$-particle absorption can be minimized by operating at high $k_{/ /}$but the values that are necessary to ensure dominant electron absorption are so large that a decrease in the overall CD efficiency and poor antenna coupling are observed. Using an antenna located at the top of the machine offers promising perspectives to avoid undesirable parasitic $\alpha$-particle heating even at low $k_{/ /}$, since for selected frequency windows the waves can be launched in-between harmonic IC resonance layers of the $\alpha$-particles and electron absorption is fully dominant. If feasible from the engineering point of view, this option offers a huge potential since the CD efficiencies obtained in this case are at least a factor of three higher than the values obtained with the standard frequency option.

\section{REFERENCES}

1. T. Franke et al., "RF systems for DEMO - challenges and opportunities", this conference

2. D.A. Ehst et al., Nuclear Fusion 31 (1991) 1933

3. D. Van Eester and R. Koch, Plasma Phys. Contr. Fusion 40 (1998) 1949

4. P.U. Lamalle, PhD thesis, 1994, LPP-ERM/KMS Report 101, Université de Mons, Mons

5. A. Messiaen et al., Nuclear Fusion 50 (2010) 025026

6. M. Nightingale et al., "FWCD technology issues for DEMO", this conference

7. D. Van Eester, E. Lerche and A. Messiaen, LPP-ERM/KMS Report 142, Brussels (2012) 